\title{
ON A CLASS OF LIPSCHITZ CONTINUOUS FUNCTIONS OF SEVERAL VARIABLES
}

\author{
TRAN DUC VAN AND NGUYEN DUY THAI SON
}

(Communicated by Andrew Bruckner)

\begin{abstract}
We establish an estimate via initial values for functions in a class of Lipschitz continuous functions of several variables. This estimate can be used to investigate the uniqueness of quasi-classical solutions of Cauchy problems for first-order nonlinear partial differential equations (PDEs). Particularly, we give an answer to an open problem posed by $\mathrm{S}$. N. Kružkov.
\end{abstract}

Let $T$ be a positive number, $\Omega_{T}=(0, T) \times \mathbb{R}^{n}=\{(t, x) \mid 0<t<T\}$, $\nabla_{x}=\left(\partial / \partial x_{1}, \partial / \partial x_{2}, \ldots, \partial / \partial x_{n}\right), n \geq 1$, and $\|\cdot\|$ and $\langle\cdot, \cdot\rangle$ be the norm and the scalar product in $\mathbb{R}^{n}$, respectively.

Denote by $\operatorname{Lip}\left(\Omega_{T}\right)$ the set of all locally Lipschitz continuous functions $u$ defined on $\Omega_{T}$. Further, set $\operatorname{Lip}\left([0, T) \times \mathbb{R}^{n}\right)=\operatorname{Lip}\left(\Omega_{T}\right) \cap C\left([0, T) \times \mathbb{R}^{n}\right)$. For every function $u$ defined on $\Omega_{T}$, we put

$$
\operatorname{Dif}(u)=\left\{(t, x) \in \Omega_{T} \mid u \text { is differentiable at }(t, x)\right\} .
$$

In this note we shall be concerned with the following class of Lipschitz continuous functions:

$$
\begin{array}{r}
V\left(\Omega_{T}\right)=\left\{u \in \operatorname{Lip}\left([0, T) \times \mathbb{R}^{n}\right) \mid \exists G \subset[0, T], \operatorname{mes}(G)=0,\right. \\
\left.\operatorname{Dif}(u) \supset \Omega_{T} \backslash\left(G \times \mathbb{R}^{n}\right)\right\} .
\end{array}
$$

In other words, a function $u \in \operatorname{Lip}\left([0, T) \times \mathbb{R}^{n}\right)$ belongs to $V\left(\Omega_{T}\right)$ iff, for almost all $t, u$ is differentiable at any point $(t, x)$.

It is obvious that

$$
\operatorname{Lip}\left([0, T) \times \mathbb{R}^{n}\right) \supset V\left(\Omega_{T}\right) \supset C^{1}\left(\Omega_{T}\right) \cap C\left([0, T) \times \mathbb{R}^{n}\right) .
$$

Our aim is to prove the following result.

Theorem 1. Let $u$ be a function in $V\left(\Omega_{T}\right)$. If there exist a nonnegative function $h$ locally bounded on $\mathbb{R}^{n}$ and a nonnegative function $k \in L^{1}(0, T)$ such that

$$
\left|\frac{\partial u(t, x)}{\partial t}\right| \leq k(t) \cdot\left[(1+\|x\|)\left\|\nabla_{x} u(t, x)\right\|+h(x)|u(t, x)|\right],
$$

Received by the editors October 19, 1992.

1991 Mathematics Subject Classification. Primary 26D10.

This work was supported in part by NCSR Vietnam Program "Applied Mathematics" and the National Basic Research Program in Natural Science. 
for almost every $t \in(0, T)$ and for all $x \in \mathbb{R}^{n}$, then

$$
|u(t, x)| \leq \exp \left[C(x) \int_{0}^{t} k(\tau) d \tau\right] . \sup _{\|y\| \leq(\|x\|+1) \exp \int_{0}^{t} k(\tau) d \tau-1}|u(0, y)|,
$$

where

$$
C(x)=\sup \left\{|h(y)| \mid\|y\| \leq(\|x\|+1) \exp \int_{0}^{T} k(\tau) d \tau-1\right\} .
$$

Corollary 1. Let $u \in V\left(\Omega_{T}\right)$ and $u(0, x) \equiv 0, x \in \mathbb{R}^{n}$. If condition (1) is satisfied for almost every $t \in(0, T)$ and for all $x \in \mathbb{R}^{n}$, then $u(t, x) \equiv 0$ in $\Omega_{T}$.

Remark 1. Corollary 1, in particular, gives the answer to problem a) posed by S. N. Kružkov in [4]. Theorem 1 and Corollary 1 can be used to investigate the uniqueness of quasi-classical solutions of the Cauchy problems for first-order nonlinear PDEs and the continuous dependence of solutions on initial conditions. In [5, 6] we obtained some results similar to Theorem 1 for subclasses of $V\left(\Omega_{T}\right)$ and used them to prove the uniqueness of global solutions of the Cauchy problems for nonlinear PDEs of first order.

Remark 2. We show by the following example that the Lipschitz continuity of $u$ is essential in Theorem 1 .

Let $J \subset[0,1]$ be the Cantor set, i.e., the set of all numbers of the form

$$
t=\sum_{i=1}^{\infty} \frac{\varepsilon_{i}}{3^{i}}
$$

where $\varepsilon_{i}$ is either 0 or 2 . The set $J$ is complete, nowhere dense on $\mathbb{R}^{1}$, and $\operatorname{mes}(J)=0$.

We define the function $v(\cdot)$, which is called the Cantor ladder, in the following way (see [3]). For $t \in J$ given by (C), we put

$$
v(t)=\sum_{i=1}^{\infty} \frac{b_{i}}{2^{i}}, \quad b_{i}=\frac{\varepsilon_{i}}{2} .
$$

If $(\alpha, \beta)$ is an open maximum interval in $(0,1) \backslash J$, then $\alpha, \beta \in J, v(\beta)=$ $v(\alpha)$. We set for $t \in(\alpha, \beta): v(t)=$ const $=v(\alpha)=v(\beta)$. It follows that $v(\cdot) \in C[0,1]$ and that $d v / d t(t)=0$ almost everywhere in $(0,1)$. In fact, $d v / d t(t)=0$ for $t \in(0,1) \backslash J$.

Putting $u(t, x)=v(t),(t, x) \in \Omega_{1}$, we easily see that

$$
\begin{gathered}
u \in C^{1}\left(((0,1) \backslash J) \times \mathbb{R}^{n}\right) \cap C\left([0,1] \times \mathbb{R}^{n}\right), \quad u(0, x) \equiv 0, \\
\partial u(t, x) / \partial t=0, \quad \forall(t, x) \in((0,1) \backslash J) \times \mathbb{R}^{n} .
\end{gathered}
$$

The function $u$ satisfies all the conditions of Theorem 1 and Corollary 1 except Lipschitz continuity. This explains why $u(t, x) \not \equiv 0$.

Proof of Theorem 1. For an arbitrary point $\left(t_{0}, x_{0}\right) \in \Omega_{T}$, we have to prove that

$$
\left|u\left(t_{0}, x_{0}\right)\right| \leq \exp \left[C\left(x_{0}\right) \int_{0}^{t_{0}} k(t) d t\right] \cdot \sup _{\|y\| \leq\left(\left\|x_{0}\right\|+1\right) \exp \int_{0}^{t_{0}} k(t) d t-1}|u(0, y)|
$$


Let $\bar{B}_{r}=\bar{B}_{r}^{n}=\left\{y \in \mathbb{R}^{n} \mid\|y\| \leq r\right\}, r \geq 0$. Denote by $\Sigma_{I}\left(t_{0}, x_{0}\right)$ the set of all absolutely continuous functions $x(\cdot): I=\left[0, t_{0}\right] \rightarrow \mathbb{R}^{n}$, which satisfy almost everywhere in $I$ the differential inclusion $d x / d t(t) \in \bar{B}_{k(t) \cdot(\|x(t)\|+1)}$ subject to the constraint $x\left(t_{0}\right)=x_{0}$.

From Theorem VI-13 in [2], it follows that $\Sigma_{I}\left(t_{0}, x_{0}\right)$ is a nonempty compact set in $C\left(I, \mathbb{R}^{n}\right)$. The sets $Z\left(t, t_{0}, x_{0}\right) \equiv\left\{x(t) \mid x(\cdot) \in \Sigma_{I}\left(t_{0}, x_{0}\right)\right\}$ and $\Gamma\left(t_{0}, x_{0}\right) \equiv\left\{(\tau, y) \mid \tau \in I, y \in Z\left(\tau, t_{0}, x_{0}\right)\right\}$ are therefore compact sets in $\mathbb{R}^{n}$ and $\mathbb{R}^{n+1}$, respectively, for all $t \in I$. Moreover, by the converse of Ascoli's theorem, the multivalued function $Z\left(\cdot, t_{0}, x_{0}\right): I \rightarrow \mathbb{R}^{n}$ is continuous.

We now define a function $\varphi(\cdot): I \rightarrow \mathbb{R}^{1}$ as

$$
\varphi(t)=\max \left\{|u(t, y)| \mid y \in Z\left(t, t_{0}, x_{0}\right)\right\} .
$$

Then, according to the maximum theorem (see [1, Theorem 1.4.16]), the fact that $u \in C\left(\Gamma\left(t_{0}, x_{0}\right)\right)$ implies $\varphi(\cdot) \in C(I)$. In addition, we have:

Lemma 1. For an arbitrary number $\theta \in\left(0, t_{0}\right), \varphi(\cdot)$ is absolutely continuous on $\left[\theta, t_{0}\right]$.

We shall also need the following:

Lemma 2. We have for every $t \in I$ the inclusion

$$
Z\left(t, t_{0}, x_{0}\right) \subset \bar{B}_{\left(\left\|x_{0}\right\|+1\right) \exp \int_{t}^{t_{0}} k(\tau) d \tau-1} .
$$

Proof of Lemma 2. For every $\eta>0$, put

$$
m_{\eta}(t)=\left(\left\|x_{0}\right\|+1+\eta\right) \exp \int_{t}^{t_{0}} k(\tau) d \tau-1 .
$$

The function $m_{\eta}(\cdot)$ is absolutely continuous, positive on $I$ with the derivative $d m_{\eta}(t) / d t=-k(t) \cdot\left(m_{\eta}(t)+1\right)$. To prove (4) we have only to show that

$$
\|x(t)\|<m_{\eta}(t), \quad \forall t \in I,
$$

for all $x(\cdot) \in \Sigma_{I}\left(t_{0}, x_{0}\right), \forall \eta>0$.

Since $m_{\eta}\left(t_{0}\right)>\left\|x_{0}\right\|=\left\|x\left(t_{0}\right)\right\|$, there exists a number $\zeta>0$ such that $m_{\eta}(t)>\|x(t)\|, \forall t \in\left(t_{0}-\zeta, t_{0}\right]$.

Assume that $(5)$ is false, so that there exists $t^{\prime} \in\left[0, t_{0}\right)$ such that $m_{\eta}\left(t^{\prime}\right) \leq$ $\left\|x\left(t^{\prime}\right)\right\|$. Putting $t_{1}=\sup \left\{t \in\left[0, t_{0}\right) \mid m_{\eta}(t) \leq\|x(t)\|\right\}<t_{0}$, we have

$$
\left\|x\left(t_{1}\right)\right\|=m_{\eta}\left(t_{1}\right), \quad m_{\eta}(t)>\|x(t)\|, \quad \forall t \in\left(t_{1}, t_{0}\right]
$$

and

$$
\begin{aligned}
d m_{\eta}(t) / d t & =-k(t) \cdot\left(m_{\eta}(t)+1\right) \leq-k(t) \cdot(\|x(t)\|+1) \\
& \leq-\|d x(t) / d t\| \leq d\|x(t)\| / d t,
\end{aligned}
$$

almost everywhere in $\left(t_{1}, t_{0}\right)$. On the other hand,

$$
\int_{t_{1}}^{t_{0}} \frac{d m_{\eta}(t)}{d t} d t>\int_{t_{1}}^{t_{0}} \frac{d\|x(t)\|}{d t} d t
$$

if and only if

$$
m_{\eta}\left(t_{0}\right)-m_{\eta}\left(t_{1}\right)=m_{\eta}\left(t_{0}\right)-\left\|x\left(t_{1}\right)\right\|>\left\|x\left(t_{0}\right)\right\|-\left\|x\left(t_{1}\right)\right\| .
$$

Hence we get a contradiction. This proves Lemma 2. 
Proof of Lemma 1. Since $u \in \operatorname{Lip}\left(\Omega_{T}\right)$, there exists $L \geq 0$ such that

$$
\begin{aligned}
&\left|u\left(t_{1}, x^{1}\right)-u\left(t_{2}, x^{2}\right)\right| \leq L\left(\left|t_{1}-t_{2}\right|+\left\|x^{1}-x^{2}\right\|\right), \\
& \forall\left(t_{1}, x^{1}\right),\left(t_{2}, x^{2}\right) \in\left(\left[\theta, t_{0}\right] \times \mathbb{R}^{n}\right) \cap \Gamma\left(t_{0}, x_{0}\right) .
\end{aligned}
$$

By the absolute continuity of the Lebesgue integral, Lemma 1 will be proved if we can show that

(6) $\left|\varphi\left(t_{1}\right)-\varphi\left(t_{2}\right)\right| \leq L\left[\left|t_{1}-t_{2}\right|+\left(\left\|x_{0}\right\|+1\right) \cdot \exp \int_{\theta}^{t_{0}} k(t) d t \cdot \int_{\left[t_{1}, t_{2}\right]} k(t) d t\right]$,

$$
\forall t_{1}, t_{2} \in\left[\theta, t_{0}\right] \text {. }
$$

Now let

$$
\varphi\left(t_{1}\right) \geq \varphi\left(t_{2}\right) \text { and } \varphi\left(t_{1}\right)=\left|u\left(t_{1}, x\left(t_{1}\right)\right)\right|,
$$

for some $x(\cdot) \in \Sigma_{I}\left(t_{0}, x_{0}\right)$. Since $x\left(t_{2}\right) \in Z\left(t_{2}, t_{0}, x_{0}\right)$, we have

$$
\begin{aligned}
0 & \leq \varphi\left(t_{1}\right)-\varphi\left(t_{2}\right)=\left|u\left(t_{1}, x\left(t_{1}\right)\right)\right|-\varphi\left(t_{2}\right) \\
& \leq\left|u\left(t_{1}, x\left(t_{1}\right)\right)\right|-\left|u\left(t_{2}, x\left(t_{2}\right)\right)\right| \leq\left|u\left(t_{1}, x\left(t_{1}\right)\right)-u\left(t_{2}, x\left(t_{2}\right)\right)\right| \\
& \leq L\left[\left|t_{1}-t_{2}\right|+\left\|x\left(t_{1}\right)-x\left(t_{2}\right)\right\|\right]=L\left[\left|t_{1}-t_{2}\right|+\left\|\int_{\left[t_{1}, t_{2}\right]} \frac{d x}{d t}(t) d t\right\|\right] \\
& \leq L\left[\left|t_{1}-t_{2}\right|+\int_{\left[t_{1}, t_{2}\right]} k(t) \cdot(\|x(t)\|+1) d t\right] .
\end{aligned}
$$

Therefore, (6) follows from Lemma 2. The proof is then complete.

Going back to the proof of Theorem 1, we put now

$$
f(t)=\int_{0}^{t} k(\tau) d \tau, \quad t \in[0, T]
$$

By Lemma 2 and the definition of $\varphi(\cdot)$, the inequality (2.a) will be obtained if we show that

$$
\varphi(t) \leq \varphi(0) \cdot \exp \left[C\left(x_{0}\right) \cdot f(t)\right], \quad \forall t \in\left[0, t_{0}\right] .
$$

For arbitrary $\mu>0$, let

$$
\psi(t)=\psi_{\mu}(t)=(\varphi(0)+\mu) \cdot \exp \left[\left(C\left(x_{0}\right)+\mu\right) \cdot(f(t)+\mu t)\right] .
$$

To get (7), we have only to prove that

$$
\varphi(t)<\psi_{\mu}(t), \quad \forall t \in\left[0, t_{0}\right] .
$$

Let $\omega=\psi-\varphi$. Then (8) is equivalent to $\omega(t)>0, \forall t \in\left[0, t_{0}\right]$. Of course, $\omega(0)=\mu>0$. We shall show that $\omega(t) \geq \omega(0), \forall t \in\left[0, t_{0}\right]$. Assume this is false, so there exists $t^{\prime} \in\left(0, t_{0}\right]$ such that $\omega\left(t^{\prime}\right)<\omega(0)$.

It is well known that there exists $G_{1} \subset(0, T), \operatorname{mes}\left(G_{1}\right)=0$ such that

$$
\frac{d f}{d t}(t)=k(t), \quad \forall t \in(0, T) \backslash G_{1} .
$$

By the hypothesis of Theorem 1, there exists $G_{2} \subset(0, T), \operatorname{mes}\left(G_{2}\right)=0$ such that $\Omega_{T} \backslash\left(G_{2} \times \mathbb{R}^{n}\right) \subset \operatorname{Dif}(u)$, and (1) holds for all $t \in(0, T) \backslash G_{2}$.

The absolute continuity of $\omega(\cdot)$ on $\left[\theta, t_{0}\right]$ implies

$$
\operatorname{mes}\left(\omega\left(G \cap\left[\theta, t_{0}\right]\right)\right)=0, \quad \forall \theta \in\left(0, t_{0}\right), \quad G=G_{1} \cup G_{2} .
$$


So

$$
\operatorname{mes}\left(\omega\left(G \cap\left[0, t_{0}\right]\right)\right)=\lim _{\theta \searrow 0} \operatorname{mes}\left(\omega\left(G \cap\left[\theta, t_{0}\right]\right)\right)=0 .
$$

From (9) and $\omega(\cdot) \in C(I)$, we could find a number $\lambda$ with

$$
\max \left\{0, \omega\left(t^{\prime}\right)\right\}<\lambda<\omega(0) \text { and } \lambda \in \omega\left[0, t^{\prime}\right] \backslash \omega\left(G \cap\left[0, t_{0}\right]\right)
$$

Let

$$
t_{*}=\inf \left\{t \in\left[0, t^{\prime}\right] \mid \omega(t)=\lambda\right\} .
$$

It is obvious that $\omega\left(t_{*}\right)=\lambda, t_{*} \in\left(0, t^{\prime}\right) \backslash G$, and that $\omega(t)>\lambda, \forall t \in\left[0, t_{*}\right)$.

Suppose that

$$
\varphi\left(t_{*}\right)=s \cdot u\left(t_{*}, x_{*}\right), \quad s=\operatorname{sign} u\left(t_{*}, x_{*}\right),
$$

for some $x_{*} \in Z\left(t_{*}, t_{0}, x_{0}\right)$; i.e., there exists a function $x^{*}(\cdot) \in \Sigma_{I}\left(t_{0}, x_{0}\right)$ so that $x^{*}\left(t_{*}\right)=x_{*}$. Choose $l \in \mathbb{R}^{n}$ with

$$
\left\langle l, s \cdot \nabla_{x} u\left(t_{*}, x_{*}\right)\right\rangle=-\left\|\nabla_{x} u\left(t_{*}, x_{*}\right)\right\|, \quad\|l\|=1 .
$$

The system of differential equations

$$
\frac{d y}{d p}(p)=(1+\|y(p)\|) \cdot l
$$

has a classical (i.e., continuously differentiable) solution on $\mathbb{R}^{1}$ satisfying the condition $y\left(f\left(t_{*}\right)\right)=x_{*}$. Let $x(t)=y(f(t)), t \in[0, T]$. Of course, $x(\cdot)$ is absolutely continuous on $[0, T], x\left(t_{*}\right)=x_{*}$, and

$$
\frac{d x}{d t}(t)=\frac{d f}{d t}(t) \cdot \frac{d y}{d p}(f(t))=k(t) \cdot(1+\|x(t)\|) \cdot l, \quad \forall t \in(0, T) \backslash G_{1} .
$$

The function ${ }_{*} x(\cdot)$ defined by

$$
{ }_{*} x(t)= \begin{cases}x(t) & \text { if } 0 \leq t \leq t_{*} \\ x^{*}(t) & \text { if } t_{*} \leq t \leq t_{0}\end{cases}
$$

belongs to $\Sigma_{I}\left(t_{0}, x_{0}\right)$. Hence,

$$
x(t) \in Z\left(t, t_{0}, x_{0}\right), \quad \forall t \in\left[0, t_{*}\right] .
$$

This implies

$$
s \cdot u(t, x(t)) \leq|u(t, x(t))| \leq \varphi(t)=\psi(t)-\omega(t)<\psi(t)-\lambda
$$

for all $t \in\left[0, t_{*}\right)$. Besides that,

$$
s \cdot u\left(t_{*}, x\left(t_{*}\right)\right)=\left|u\left(t_{*}, x_{*}\right)\right|=\varphi\left(t_{*}\right)=\psi\left(t_{*}\right)-\omega\left(t_{*}\right)=\psi\left(t_{*}\right)-\lambda .
$$

Since $t_{*} \in(0, T) \backslash G$, we see that:

(i) $u$ is differentiable at $\left(t_{*}, x_{*}\right)$,

(ii) $x(\cdot)$ is differentiable at $t_{*}$ with $d x / d t\left(t_{*}\right)=k\left(t_{*}\right) \cdot\left(1+\left\|x_{*}\right\|\right) \cdot l$,

(iii) $\psi(\cdot)$ is differentiable at $t_{*}$ with

$$
\frac{d \psi}{d t}\left(t_{*}\right)=\left(C\left(x_{0}\right)+\mu\right) \cdot\left(k\left(t_{*}\right)+\mu\right) \cdot \psi\left(t_{*}\right) .
$$


So it follows from (11), (12) that

$$
\left.\frac{d}{d t}[s \cdot u(t, x(t))]\right|_{t=t_{*}} \geq \frac{d \psi}{d t}\left(t_{*}\right) .
$$

Consequently,

$$
\begin{gathered}
s \cdot \frac{\partial u}{\partial t}\left(t_{*}, x\left(t_{*}\right)\right)+\left\langle d x / d t\left(t_{*}\right), s \cdot \nabla_{x} u\left(t_{*}, x\left(t_{*}\right)\right)\right\rangle \\
\geq\left(C\left(x_{0}\right)+\mu\right) \cdot\left(k\left(t_{*}\right)+\mu\right) \cdot \psi\left(t_{*}\right) .
\end{gathered}
$$

Hence,

$$
\begin{gathered}
s \cdot \frac{\partial u}{\partial t}\left(t_{*}, x_{*}\right)+k\left(t_{*}\right)\left(1+\left\|x_{*}\right\|\right) \cdot\left\langle l, s \cdot \nabla_{x} u\left(t_{*}, x_{*}\right)\right\rangle \\
\geq\left(C\left(x_{0}\right)+\mu\right) \cdot\left(k\left(t_{*}\right)+\mu\right) \cdot\left(\left|u\left(t_{*}, x_{*}\right)\right|+\lambda\right) .
\end{gathered}
$$

Because $\mu>0$ and $\lambda>0$, the last inequality implies that

$$
\left|\frac{\partial u}{\partial t}\left(t_{*}, x_{*}\right)\right|>k\left(t_{*}\right) \cdot\left[\left(1+\left\|x_{*}\right\|\right) \cdot\left\|\nabla_{x} u\left(t_{*}, x_{*}\right)\right\|+C\left(x_{0}\right) \cdot\left|u\left(t_{*}, x_{*}\right)\right|\right] \text {. }
$$

Clearly, (13) contradicts (1), which shows that there could not exist any $t^{\prime} \in$ $\left[0, t_{0}\right]$ with $\omega\left(t^{\prime}\right)<\omega(0)$. So

$$
\omega(t) \geq \omega(0)>0 \text { for all } t \in\left[0, t_{0}\right]
$$

therefore, (8) is proved. This completes the proof of Theorem 1.

\section{REFERENCES}

1. J.-P. Aubin and H. Frankowska, Set-valued analysis, Birkhäuser, Basel, 1990.

2. Ch. Castaing and M. Valadier, Convex analysis and measurable multifunctions, Lecture Notes in Math., vol. 580, Springer-Verlag, New York, 1977.

3. J. Dieudonné, Foundations of modern analysis, Academic Press, New York, 1960.

4. S. N. Kružkov, Theory of functions and differential equations, Vestnik Moskov. Univ. Ser. I Mat. Mekh. 5 (1991), 36-43. (Russian)

5. T. D. Van and N. D. T. Son, On the uniqueness of global classical solutions of the Cauchy problem for Hamilton-Jacobi equations, Acta Math. Vietnam. 17 (1992), 161-167.

6. __ Uniqueness of global quasi-classical solutions of the Cauchy problem for the equation $\partial u / \partial t+(\partial u / \partial x)^{2}=0$, Tạp chí Toán học 19 (1991), 65-71.

Hanoi Institute of Mathematics, Box 631, Boho, Hanol, Vietnam 